\title{
Light-front field theory in the description of hadrons
}

\author{
Chueng-Ryong $\mathrm{Ji}^{1, \mathrm{a}}$ \\ ${ }^{1}$ Department of Physics, Box 8202, North Carolina State University, Raleigh, NC 27695-8202
}

\begin{abstract}
We discuss the use of light-front field theory in the descriptions of hadrons. In particular, we clarify the confusion in the prevailing notion of the equivalence between the infinite momentum frame and the light-front dynamics and the advantage of the lightfront dynamics in hadron physics. As an application, we present our recent work on the flavor asymmetry in the proton sea and identify the presence of the delta-function contributions associated with end-point singularities arising from the chiral effective theory calculation. The results pave the way for phenomenological applications of pion cloud models that are manifestly consistent with the chiral symmetry properties of QCD.
\end{abstract}

\section{Introduction}

When particle systems have characteristic momenta which are of the same order or even much larger than the masses of the particles involved, it is part of nature that a relativistic treatment is called for in order to describe those systems properly. In particular, relativistic effects are most essential to describe the low-lying hadron systems in terms of strongly interacting quarks/antiquarks and gluons in quantum chromodynamics (QCD). For the study of relativistic particle systems, Dirac[1] proposed three different forms of the relativistic Hamiltonian dynamics in 1949: i.e. the instant $\left(x^{0}=0\right)$, front $\left(x^{+}=\left(x^{0}+x^{3}\right) / \sqrt{2}=0\right)$, and point $\left(x_{\mu} x^{\mu}=a^{2}>0, x^{0}>0\right)$ forms. The instant form dynamics (IFD) of quantum field theories is based on the usual equal time $t=x^{0}$ quantization (units such that $c=1$ are taken here) and provides a traditional approach evolved from non-relativistic dynamics. The IFD makes a close contact with Euclidean space, developing temperature-dependent quantum field theory, lattice QCD, etc. The equal light-front time $\tau \equiv(t+z / c) / \sqrt{2}=x^{+}$quantization yields the front form dynamics, nowadays more commonly called light-front dynamics (LFD) and provides a progressive approach for the study of relativistic dynamics. The LFD works strictly in Minkowski space, developing useful frameworks for the analyses of deep inelastic scattering (DIS), parton distribution functions (PDFs), deeply virtual Compton scattering (DVCS), generalized parton distributions (GPDs), etc. The quantization in the point form $\left(x^{\mu} x_{\mu}=a^{2}>0, x^{0}>0\right)$ is called radial quantization and this quantization procedure has been much used in string theory and conformal field theories [2] as well as in hadron physics [3-5]. Among these three forms of relativistic dynamics proposed by Dirac, the LFD carries the largest number (seven) of the kinematic (or interaction independent) generators leaving the least number (three) of the dynamics generators while both the IFD and the point form dynamics carry six kinematic generators and four dynamic generators within the total ten Poincaré generators. Indeed, no matter what form of relativistic dynamics is taken, the number of kinematic generators

ae-mail: crji@ncsu.edu 


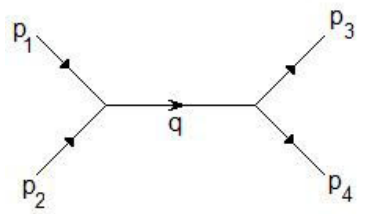

Figure 1. Scattering amplitude of spinless particles.

cannot be larger than seven, i.e. the LFD has this maximum number of kinematic generators. Thus, the LFD maximizes the capacity to save dynamical efforts in obtaining the QCD solutions that reflect the full Poincaré symmetries. In this presentation, we discuss this remarkable advantage of the LFD in hadron physics and exemplify it in the study of the flavor asymmetry in the proton sea.

\section{Interpolating Scattering Amplitudes}

The IFD and the LFD can be interpolated[6-10] by an interpolation angle between the ordinary time $t$ and the light-front time $\tau$. Introducing the interpolating scattering amplitude[8-10] that links the corresponding time-ordered amplitudes between the two forms of dynamics, we discussed the physical meaning of the kinematic transformations in contrast to the dynamic transformations by means of checking the invariance of each individual time-ordered amplitude for an arbitrary interpolation angle. Using this interpolation method, we were able to also trace a dramatic change of the longitudinal boost's characteristics, namely, from "dynamic" in IFD to "kinematic" in LFD. This provides a distinct advantage to LFD in hadron phenomenology.

To trace the forms of relativistic quantum field theory between IFD and LFD, we begin by adopting the following convention of the space-time coordinates to define the interpolation angle[6-10]:

$$
\left[\begin{array}{l}
x^{\mp} \\
x^{-}
\end{array}\right]=\left[\begin{array}{cc}
\cos \delta & \sin \delta \\
\sin \delta & -\cos \delta
\end{array}\right]\left[\begin{array}{l}
x^{0} \\
x^{3}
\end{array}\right],
$$

in which the interpolation angle is allowed to run from 0 through $45^{\circ}, 0 \leq \delta \leq \frac{\pi}{4}$. All the indices with the wide-hat notation signify the variables with the interpolation angle $\delta$. For the limit $\delta \rightarrow 0$ we have $x^{\bar{\mp}}=x^{0}$ and $x^{\bar{\mp}}=-x^{3}$ so that we recover usual space-time coordinates although the $\mathrm{z}$-axis is inverted while for the other extreme limit, $\delta \rightarrow \frac{\pi}{4}$ we have $x^{\mp}=\left(x^{0} \pm x^{3}\right) / \sqrt{2} \equiv x^{ \pm}$which leads to the standard light-front coordinates. Of course, the same interpolation applies to the momentum variables.

For the simplest possible illustration of applying the interpolation to relativistic quantum field theory[8], we first discuss the scattering amplitude of two spin-less particles, i.e., the analogue of the well known QED annihilation/production process $e^{+} e^{-} \rightarrow \mu^{+} \mu^{-}$in a toy $\phi^{3}$ model theory, as depicted in Fig.1. As shown in Refs.[9, 10], we have extended our discussion to the gauge bosons and the fermion spinors introducing the interpolating helicity of polarization vectors and spinors. However, for the simplicity of our discussion in this presentation, we don't involve spins or any other dynamical degrees of freedom beyond the momenta of particles to discuss just the main point. Modulo inessential factors including the square of the coupling constant, the lowest order tree-level Feynman diagram shown in Fig. 1 is proportional to the propagator of the intermediate particle: $\Sigma=1 /\left(s-m^{2}\right)$, where $s=\left(p_{1}+p_{2}\right)^{2}$ is the Mandelstam variable, which is invariant under any Poincaré transformations, and $m$ is the mass of the intermediate boson. Of course, the physical process can take place only 


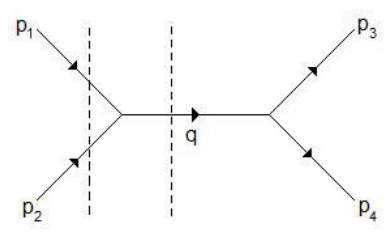

(a)

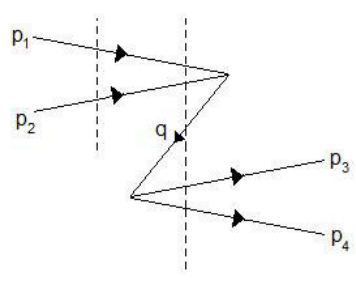

(b)

Figure 2. Time-ordered amplitudes for the Feynman amplitude depicted in Fig.1.

above the threshold $s>4 M^{2}$, where $M$ is the mass of the final particle and anti-particle that are produced, e.g. the muon mass in the $e^{+} e^{-} \rightarrow \mu^{+} \mu^{-}$scattering process. The corresponding timeordered amplitudes for an arbitrary interpolation angle $\delta$ are graphically represented in Fig.2(a) and Fig.2(b) and respectively given by

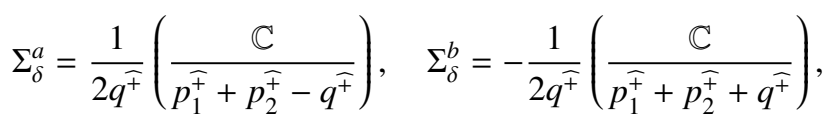

where $\mathbb{C}=\cos 2 \delta$. We note that the individual time-ordered amplitudes are invariant only under the transformations given by the kinematic generators, i.e. they are not invariant under the transformations given by the dynamic generators, although the sum of the time-ordered amplitudes, e.g. the Feynman diagram shown in Fig.1, is invariant under all ten Poincaré generators. This discussion clarifies the physical meaning of the kinematic generators vs. the dynamic generators.

Fig. 3 shows $\Sigma_{\delta}^{a}$ and $\Sigma_{\delta}^{b}$ as functions of the initial particle's total momentum $\left(\vec{p}_{1}+\vec{p}_{2}\right) \cdot \hat{z}=P_{z}$ while $\left(\vec{p}_{\hat{1}}+\vec{p}_{\hat{2}}\right) \cdot \hat{x}=0$ and $\left(\vec{p}_{\hat{1}}+\vec{p}_{\hat{2}}\right) \cdot \hat{y}=0$, and the interpolation angle $\delta$. The ranges of $\delta$ and $P_{z}$ are taken as $0 \leq \delta<\frac{\pi}{4}$ and $-4 \leq P_{z} \leq 4$ in some unit of energy, e.g. GeV, respectively. For illustrative purpose, we took $s=2$ and $m=1$ using the same energy unit.

(a)

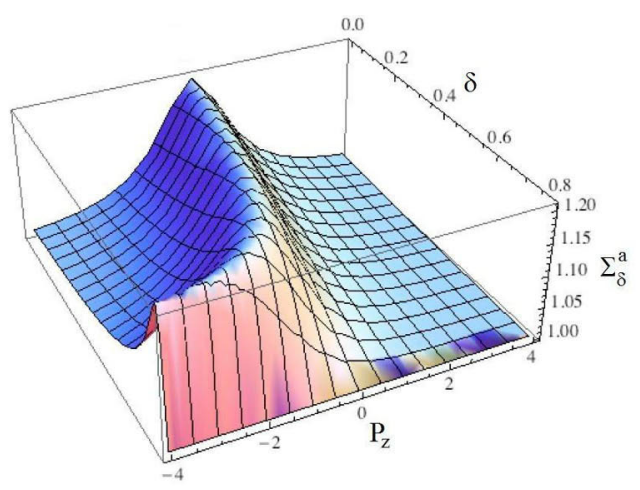

Figure 3. Interpolating Amplitudes

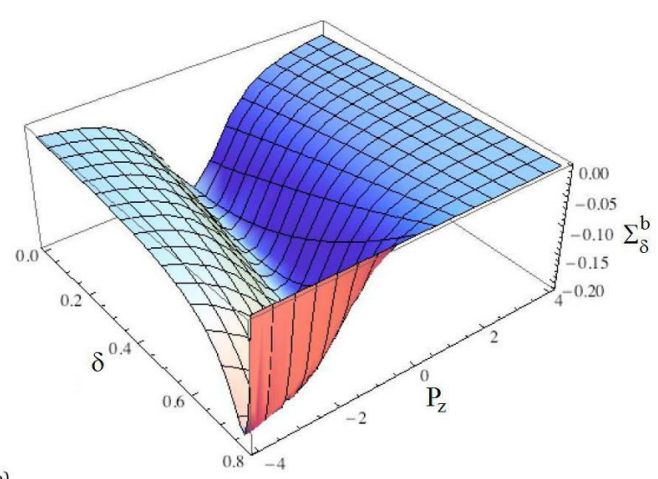

(b) 
As clearly visible in Fig. 3, the contributions from $\Sigma_{\delta}^{a}$ and $\Sigma_{\delta}^{b}$ are such that the sum of them yields a constant, independent of $P_{z}$ and $\delta$. For $\delta=0, \Sigma_{\delta}^{a}$ and $\Sigma_{\delta}^{b}$ are the maximal and minimal, respectively, at $P_{z}=0$. For $\delta=\frac{\pi}{4}, \Sigma_{\delta}^{a}$ is the whole answer and $\Sigma_{\delta}^{b}=0$. The individual time-ordered amplitudes, $\Sigma_{\delta=\pi / 4}^{a}$ and $\Sigma_{\delta=\pi / 4}^{b}$, are now separately independent of $P_{z}$ or invariant under the longitudinal boost. This shows clearly that the longitudinal boost becomes the kinematic generator in LFD and makes the number of kinematic generators get maximized in LFD.

We now pay attention to the apparent $J$-shaped ridge in $\Sigma_{\delta}^{a}$ matched by a similar valley in $\Sigma_{\delta}^{b}$. For positive values of momentum, $P_{z}>0$, the amplitudes $\Sigma_{\delta}^{a}$ and $\Sigma_{\delta}^{b}$ show a smooth behaviour, while for negative values of $P_{z}$ we observe the presence of the $J$-shaped curve. We find that these $J$-shaped features are given by the function $P_{z}=-\sqrt{\frac{s(1-\mathbb{C})}{2 \mathbb{C}}}$. An interesting point to observe in this $J$-shaped curve for negative values of momentum $P_{z}$ is that it is stable as it is independent of the mass and does not disappear as the momentum goes to the negative infinity. Thus, if the limit $\delta \rightarrow \frac{\pi}{4}$ is taken in the exact amplitude with $P_{z}$ given by the $J$-shaped curve, i.e. $P_{z}=-\sqrt{\frac{s(1-\mathbb{C})}{2 \mathbb{C}}} \stackrel{\mathbb{C} \rightarrow 0}{\longrightarrow}-\infty$, then the connected contribution to the current arising from the vacuum $\Sigma_{\delta \rightarrow \frac{\pi}{4}}^{b}$ does not vanish but remains as a nonzero constant, i.e., $-\frac{1}{2 m(\sqrt{s}+m)}=-\frac{1}{2(\sqrt{2}+1)} \approx-0.207$, although this nonzero constant (i.e. the minimum of $\left.\Sigma_{\delta \rightarrow \frac{\pi}{4}}^{b}\right)$ is cancelled by the overshoot in the maximum of $\Sigma_{\delta \rightarrow \frac{\pi}{4}}^{a}$, given by $\frac{1}{2 m(\sqrt{s}-m)}=\frac{1}{2(\sqrt{2}-1)} \approx 1.207$ to yield the total amplitude $\frac{1}{s-m^{2}}=1$.

Here, one may notice that the limits $P_{z} \rightarrow-\infty$ and $\delta \rightarrow \frac{\pi}{4}$ do not commutate. However, this fact should not be construed as an ambiguity of the suggested interpolation procedure, which is completely independent of the order of taking these limits. Rather, one may understand the non-commutativity as a possible issue for the common folklore of "equivalence of IFD and LFD in the infinite momentum frame (IMF)". Our work qualifies this notion, since it applies in the limit of $P_{z} \rightarrow \infty$, but requires a great caution in the limit $P_{z} \rightarrow-\infty$, i.e. $P^{+}=\left(P^{0}+P_{z}\right) / \sqrt{2} \rightarrow 0$ limit. One should note that, as $P^{+}=p_{1}^{+}+p_{2}^{+} \rightarrow 0$, both $p_{1}^{+} \rightarrow 0$ and $p_{2}^{+} \rightarrow 0$, i.e. the light-front plus (+) momenta of all the particles involved in the scattering become zero. Thus, only the light-front zero-modes contribute to the scattering amplitude in this limit. This is remarkable because the limits from the IMF[11] of IFD to LFD are clearly asymmetric between $P^{+} \rightarrow \infty$ and $P^{+} \rightarrow 0$, while the IMF of IFD itself is entirely symmetric between $P_{z} \rightarrow \infty$ and $P_{z} \rightarrow-\infty$. In the interpolation we discuss here, the treacherous point is related to the particular way the limit $P_{z} \rightarrow-\infty$ is taken. If it is taken along the J-shaped curve, i.e., $P_{z}=-\sqrt{\frac{s(1-\mathbb{C})}{2 \mathbb{C}}} \stackrel{\mathbb{C} \rightarrow 0}{\longrightarrow}-\infty$, then the result $\Sigma_{\delta=\frac{\pi}{4}}^{b}=-\frac{1}{2 m(\sqrt{s}+m)} \neq 0$ is valid, but if $\delta$ and $P_{z}$ are not so correlated that the longitudinal boost invariance of the LFD is manifest then $\Sigma_{\delta=\frac{\pi}{4}}^{b}=0$. No matter how the limits turn out, one should note that the longitudinal boost is kinematic in LFD and the invariance of each time-ordered amplitude must hold under the transformations given by the kinematic generators. The sum of the two amplitudes $\Sigma_{\delta=\frac{\pi}{4}}^{a}+\sum_{\delta=\frac{\pi}{4}}^{b}$ remains of course invariant as it should be.

Although we discussed here the simplest possible scattering amplitude of two spinless particles in a toy $\phi^{3}$ model theory, it clarified the confusion in the prevailing notion of the equivalence between the infinite momentum frame and the LFD. It also alerts the caution in handling the contribution from the light-front zero-modes.

\section{Leading Nonanalytic Behavior in Chiral Dynamics}

While the light-front zero-modes appear treacherous as discussed above, they play a vital role in guiding the hadron phenomenology to fulfill the first principles in hadron physics. As an example, we 
discuss here the leading nonanalytic (LNA) behavior determined by the infrared properties of chiral loops (characteristic of Goldstone boson loops in chiral perturbation theory[12]). The LNA behavior in chiral dynamics is model-independent[13-15], and places the physics of the pion cloud on a firm ground in QCD.

The pion cloud of the nucleon plays a vital role in understanding the nucleon's long-range structure as it is known from the approximate chiral symmetry of QCD. It provides important corrections to static nucleon properties, such as the mass, magnetic moment and axial charge, and significantly influences its electric and magnetic charge distributions (for a review see Ref.[16]). This will also have important consequences for correctly implementing chiral extrapolation of lattice QCD data on parton distribution moments and subsequently on other quantities, such as GPDs[17], as well as on understanding the pion distributions in the nucleon in a well-defined theoretical framework. In addition to the traditional studies of elastic form factors and parton distributions, there has also been a great deal of interest in GPDs. As with the ordinary PDFs, the physical interpretation of GPDs in terms of probability distributions is most natural in the LFD, and one knows that here too chiral corrections can be very important. It is timely, therefore, to address the question of how to provide a consistent derivation of the chiral corrections to all of these observables in LFD.

We have recently applied the LFD methodology to the case of pion loops and their effects on the $\bar{d}-\bar{u}$ asymmetry in the proton [18]. The observation of the $\bar{d}-\bar{u}$ flavor asymmetry in the light quark sea of the proton [19-22] has been one of the seminal results in hadronic physics of the past two decades, leading to a major reevaluation of our understanding of the quark structure of the nucleon. In particular, the measurement revealed the importance of 5-quark Fock state components of the nucleon's light-front wave function, and the crucial role played by chiral symmetry breaking and the pion cloud in describing the nucleon's internal structure. An important development in establishing a formal link between models of $\bar{d}-\bar{u}$ and QCD came with the realization that the moments of PDFs could be formally expanded in chiral perturbation theory $(\chi \mathrm{PT})$ in terms of power series in the pion mass squared, $m_{\pi}^{2}$. In particular, the LNA contributions were found to depend on the (model-independent) long range structure of the pion cloud, with a characteristic $m_{\pi}^{2} \log m_{\pi}^{2}$ dependence [12], whose coefficient is given by physical constants [14]. This idea was then applied to the chiral extrapolation of lattice QCD moments of the nonsinglet $u-d$ distribution in order to reconcile the lattice data at large pion masses with experiment [13]. We recently refined the computation [23] of the $\bar{d}-\bar{u}$ flavor asymmetry in the proton sea with a complementary effort to reveal the dynamics of pion exchange in high-energy processes and generalized our approach to the SU(3) sector [24]. Before we discuss these development, we present in this section the role of the light-front zero-modes in obtaining the model-independent LNA coefficient in chiral dynamics using a simple heuristic example first.

To discuss a treacherous point involved in LFD, one may look into a simple example of twodimensional loop amplitude for a particle of mass $m$ :

$$
I=\int d^{2} k \frac{1}{k^{2}-m^{2}+i \epsilon} .
$$

In the covariant approach, this amplitude can be computed by dimensional regulation and is given by

$$
I=-i \pi\left(\frac{2}{2-d}-\log \pi-\gamma+O(2-d)-\log \frac{m^{2}}{\mu^{2}}\right),
$$

where $\mu$ is a mass parameter introduced to give the correct mass dimensions in $d$ dimensions, $\gamma \approx$ 0.577 is Euler's constant, and $d$ goes to 2 in this example. The LNA behavior of this amplitude in the limit $m \rightarrow 0$ is thus given by $I_{\mathrm{LNA}}=i \pi \log m^{2}$. In time-ordered perturbation theory, it is 
straightforward to use Cauchy's integral theorem and compute the amplitude as follows:

$$
I=\int d k^{1} d k^{0} \frac{1}{\left(k^{0}+\omega_{k}-i \epsilon\right)\left(k^{0}-\omega_{k}+i \epsilon\right)}=-2 i \pi \int_{0}^{\Lambda \rightarrow \infty} d k^{1} \frac{1}{\sqrt{\left(k^{1}\right)^{2}+m^{2}}}=\lim _{\Lambda \rightarrow \infty} 2 i \pi \log \frac{m}{2 \Lambda}
$$

where $\omega_{k}=\sqrt{\left(k^{1}\right)^{2}+m^{2}}$ is the on-mass-shell energy of the particle and $\Lambda$ is the cutoff parameter in the momentum integration of $k^{1}$. The LNA behavior of this amplitude is identical to $I_{\mathrm{LNA}}=i \pi \log m^{2}$. However, the LFD calculation of this amplitude is not so simple due to a dramatic change of pole structure:

$$
I=\frac{1}{2} \int d k^{+} d k^{-} \frac{1}{k^{+} k^{-}-m^{2}+i \epsilon}=\frac{1}{2} \int \frac{d k^{+}}{k^{+}} \int d k^{-} \frac{1}{k^{-}-\frac{m^{2}}{k^{+}}+i \frac{\epsilon}{k^{+}}},
$$

where the pole $\left(\mathrm{m}^{2} / k^{+}-i \epsilon / k^{+}\right)$of the LF energy $k^{-}$depends on the $k^{+}$variable. Not only is there just one pole in the $k^{-}$plane but also the $k^{-}$pole is moving as $k^{+}$varies. One should realize that neither $k^{+}>0$ nor $k^{+}<0$ can yield any nontrivial contribution as one can take a corresponding contour in each region $\left(k^{+}>0\right.$ or $\left.k^{+}<0\right)$ to get the zero result $(I=0)$ according to the Cauchy's integral theorem. The only non-trivial contribution is attained from the light-front zero-mode $k^{+}=0$. It is crucial to capture the runaway pole at $k^{+}=0$ in order to get the correct result. The details of this treacherous point have been discussed in Ref.[25]. To capture the zero-mode contribution, i.e. the runaway $k^{+}=0$ pole, we used the LF cylindrical coordinates $\left(k^{+}=r \cos \phi, k^{-}=r \sin \phi\right)$ to perform the $k^{+}$and $k^{-}$integration as follows:

$$
I=\frac{1}{2} \int_{0}^{\infty} d r r \int_{0}^{2 \pi} d \phi\left(r^{2} \sin \phi \cos \phi-m^{2}+i \epsilon\right)^{-1}=\lim _{R \rightarrow \infty}\left(i \pi \log \frac{2 m^{2}}{R^{2} e^{-i \pi / 2}}+O\left(1 / R^{4}\right)\right),
$$

where $R$ is the cutoff parameter in the LF cylindrical coordinate integration. The result correctly yields $I_{\mathrm{LNA}}=i \pi \log m^{2}$, identical to the results of the covariant and equal-time calculations. It demonstrates the vital role of the light-front zero-mode to obtain the correct LNA coefficient in this heuristic example. Initial calculations of pion loop effects were performed in the context of the "Sullivan" process [26], using pseudoscalar (PS) pion-nucleon coupling, which involves only the pion "rainbow" diagram. Analysis within the $\chi \mathrm{PT}$, which implements the chirally symmetric pseudovector (PV) coupling, reveals differences in the off-shell behavior of the loops [27], as well as additional pion bubble terms $[14,15]$ which contribute only at the light-front zero-mode $k^{+}=0$ [28]. The equivalence between the PS and PV theories was recently discussed in detail in Refs. [28, 29]. This equivalence between the PV and PS theories may be summarized in Fig.4. In this description, the first line shows the equivalence between PV and PS for which it is crucial to include the contribution from the chiral partner that corresponds to the two-pion contribution, a la $\sigma \bar{N} N \sim \pi \pi \bar{N} N$, depicted as the white blob of the vertex in the tadpole diagram shown in first line of Fig.4. This equality can be verified straightforwardly using the Dirac equation for the external nucleons [30]. Moreover, as depicted by the two parallel downward arrows in Fig. 4, the fermion propagator can be split into the so-called "on-shell" contribution plus the LF instantaneous contribution [31]: i.e.

$$
\frac{1}{q-m}=\frac{\sum_{s} u(q, s) \bar{u}(q, s)}{q^{2}-m^{2}}+\frac{\gamma^{+}}{2 q^{+}}
$$

where $q$ is the four-momentum of the propagating fermion. We see here that both the instantaneous contribution and the tadpole diagram contribution are the light-front zero-mode contributions proportional to $\delta\left(k^{+}\right)$. As shown in Fig. 4, these two zero-mode contributions (tadpole type diagram and instantaneous contribution) cancels each other in the PS theory while the instantaneous contribution 


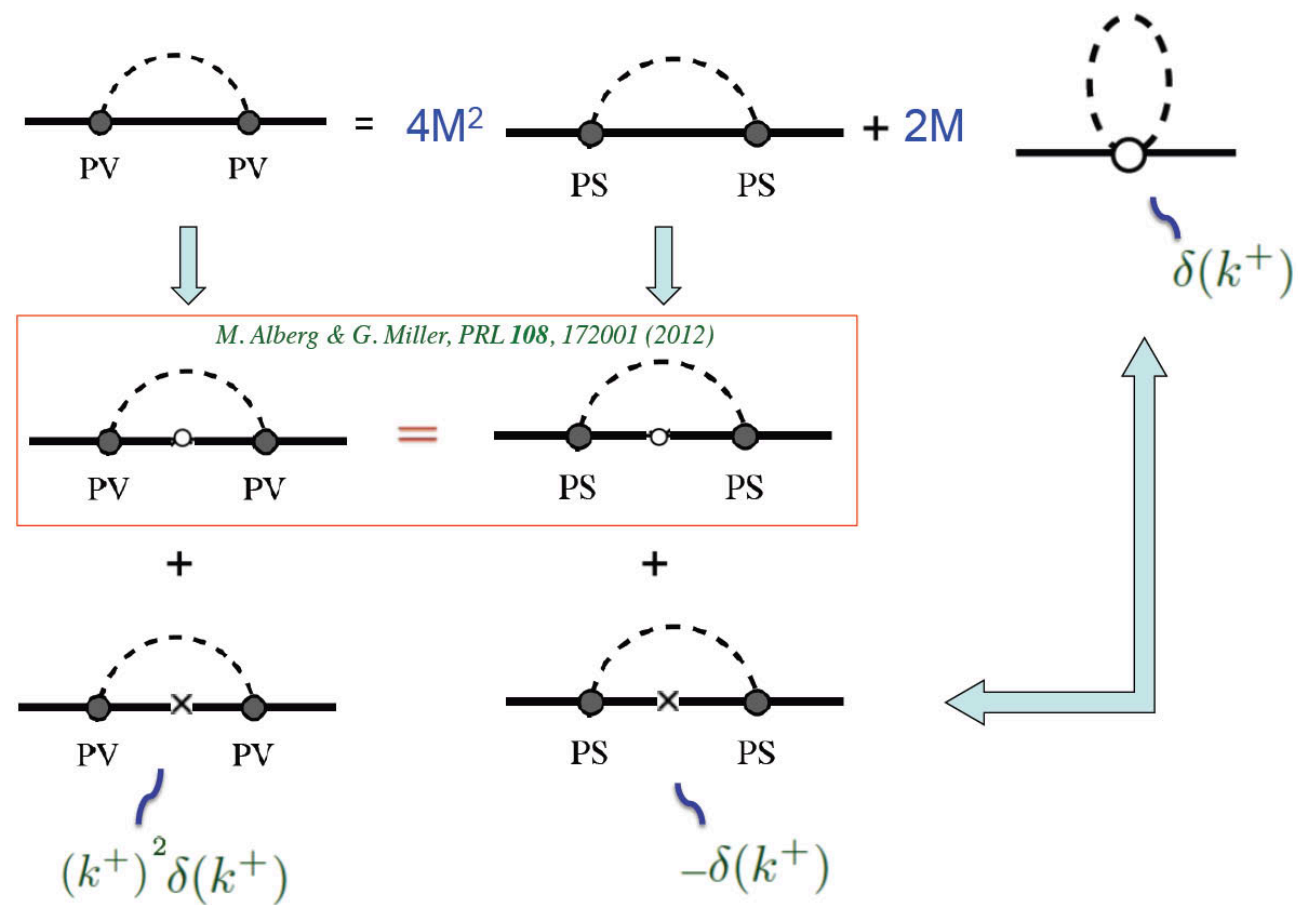

Figure 4. The equivalence between the pseudovector (PV) coupling and the pseudoscalar (PS) coupling in chiral dynamics. The chiral partner in the PS theory corresponds to the two-pion coupling denoted by the large white blob in the upper rightmost (tadpole type) diagram. The two parallel downward arrows represent the equality of the fermion propagator given by Eq.(8). The equivalence in the "on-shell" contribution between PV and PS coupling theories is boxed with Ref.[32]. The cancellation between the two light-front zero-mode contributions in the PS coupling is denoted by the double-arrow pointing to those two contributions, i.e. the chiral partner contribution and the instantaneous contribution.

in the PV theory vanishes by itself due to the derivative coupling. Thus, one may effectively get the equivalence between the "on-shell" contributions of the PV and PS theories in the self-energy calculation [32]. This also confirms the model-independence of the LNA behavior in the nucleon self-energy regardless whether the PV theory or the PS theory is used. However, it is still crucial to keep track of the light-front zero-modes as their finite nonzero contribution exists in other process such as the vertex correction [27]. The heuristic example discussed earlier has already shown the crucial role of the light-front zero-mode contribution. We have indeed verified the chiral symmetry of QCD in the $\bar{d}-\bar{u}$ asymmetry in the proton [18] by examining the LNA behavior of the lowest moment $\bar{D}-\bar{U} \equiv \int_{0}^{1} d x(\bar{d}-\bar{u})$. The LNA behavior of $\bar{D}-\bar{U}$ is given by

$$
\begin{aligned}
(\bar{D}-\bar{U})_{\mathrm{LNA}} & =\frac{3 g_{A}^{2}+1}{2\left(4 \pi f_{\pi}\right)^{2}} m_{\pi}^{2} \log m_{\pi}^{2} \\
& =\frac{4 g_{A}^{2}+\left(1-g_{A}^{2}\right)}{2\left(4 \pi f_{\pi}\right)^{2}} m_{\pi}^{2} \log m_{\pi}^{2}
\end{aligned}
$$




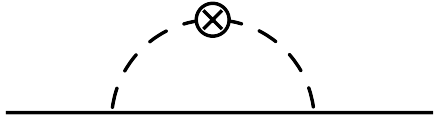

(a)

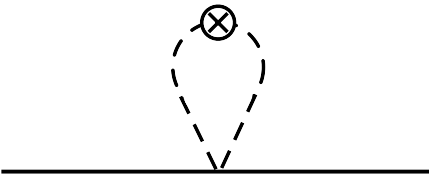

(b)

Figure 5. Contributions to the pion light-cone momentum distributions in the nucleon, from (a) the pion rainbow, and (b) the pion bubble diagrams.

where $g_{A}=1.267$ is the axial charge and $f_{\pi}=93 \mathrm{MeV}$ is the pion decay constant. In the second line of this equation, Eq.(9), the model-independent LNA coefficient $3 g_{A}^{2}$ is explicitly split into the "on-shell" contribution $4 g_{A}^{2}$ and the light-front zero-mode ( $\delta$-function) contribution $-g_{A}^{2}$. This result in the second line of Eq.(9) resolved [28, 29] the factor 4/3 difference between the "Sullivan" process calculation [12] and the $\chi \mathrm{PT}$ calculation $[14,15]$, which has been puzzling for more than a decade. In fact, one can now realize that the absence of the $\delta$-function contributions in the PS version of the rainbow diagrams leads to the coefficient of the leading nonanalytic term in the chiral expansion of the quark distribution function moments that differs by a factor $4 / 3$ from the PV result. Thus, in this resolution of the lingering factor difference, it is evident that $\delta$-function contribution from the light-front zero-mode has been crucial.

\section{Flavor asymmetry in proton sea}

The model-independence of the LNA behavior in chiral dynamics is so useful that one may regard it as a sort of global positioning system "GPS" in the practice of chiral phenomenology. With this guidance, we recently refined the computation [23] of the $\bar{d}-\bar{u}$ flavor asymmetry in the proton sea with a complementary effort to reveal the dynamics of pion exchange in high-energy processes and generalized our approach to the SU(3) sector [24]. This complementary effort has been the study of leading neutron production in semi-inclusive DIS on the proton, in which a forward moving neutron is produced in coincidence with the scattered lepton in the high-energy reaction $e p \rightarrow e n X$. Several dedicated experiments at the ep collider HERA [33-35] have collected high-precision data on the spectrum of leading neutrons carrying a large fraction of the proton's energy. Moreover, the upcoming tagged DIS (TDIS) experiment at Jefferson Lab [36] plans to take data on the production of leading protons from an effective neutron target in the reaction $e n \rightarrow e p X$, which, in analogy with the HERA leading neutron leptoproduction, can be described through the exchange of a $\pi^{-}$. In the proposed experiment, the effective neutron target will be prepared by tagging spectator protons with momenta between $60 \mathrm{MeV}$ and $400 \mathrm{MeV}$ at backward kinematics in the DIS of the electron from a deuteron nucleus, using the same technique that was developed for the measurement of the neutron structure function in the BONuS experiment at Jefferson Lab [37].

In particular, we have recently addressed [23] the question of whether one can reduce the model dependence of $F_{2}^{\pi}$ extracted from the HERA data [33-35] by using additional constraints from the data on the SU(2) flavor asymmetry $\bar{d}-\bar{u}$, especially those from the E866 Drell-Yan experiment [22]. Because the E866 data are at relatively high $x$ values compared with the HERA measurements, within the pion exchange framework they are sensitive to the pion PDFs at large $x_{\pi}$, where these are well determined from pion-nucleon Drell-Yan data [38-40]. The main variable in describing the $\bar{d}-\bar{u}$ 
asymmetry is therefore the pion distribution function in the nucleon. In our study [23], we used methodology adopted from global PDF analysis [41, 42] to simultaneously fit both the HERA leading neutron and E866 $\bar{d}-\bar{u}$ asymmetry data. This study allowed us to refine the analysis of the $\bar{d}-\bar{u}$ asymmetry and at the same time discuss a possible prediction of the future tagged DIS experiments in JLab.

\section{Conclusion and future directions}

The LFD is not just formal but consequential in the analysis of physical observables. The interpolation method that we apply for the scattering amplitude was instrumental to observe the dramatic change of the longitudinal boost's character from dynamic in IFD to kinematic in LFD. It clarified also the confusion in the prevailing notion of the equivalence between the IMF of IFD and the LFD. Maximizing the number of kinematic generators in LFD is a great advantage in saving dynamic efforts to obtain the QCD solutions that reflect the full Poincare symmetries. While the light-front zero-mode is treacherous, it plays a vital role in resolving theoretical issues as we have discussed the lingering LNA factor difference in chiral dynamics which has been persistent more than a decade. The model-independence of the LNA behavior in chiral dynamics provides a good guidance in chiral phenomenology. More recently, we generalized this approach to the SU(3) sector [24]. We performed a comprehensive analysis of the strange-antistrange parton distribution function (PDF) asymmetry in the proton in the framework of chiral effective theory, including the full set of lowest-order kaon loop diagrams with off-shell and contact interactions, in addition to the usual on-shell contributions previously discussed in the literature. We identified the presence of $\delta$-function contributions to the $\bar{s}$ PDF at $x=0$, with a corresponding valencelike component of the $s$-quark PDF at larger $x$, which allows greater flexibility for the shape of $s-\bar{s}$. Expanding the moments of the PDFs in terms of the pseudoscalar kaon mass, we computed the leading nonanalytic behavior of the number and momentum integrals of the $s$ and $\bar{s}$ distributions, consistent with the chiral symmetry of QCD. We discussed the implications of our results for the understanding of the NuTeV anomaly [43] and for the phenomenology of strange-quark PDFs in global QCD analysis. However, the quantitative analysis of the light-front zero-mode contribution in the hadron phenomenology is still open for the progress.

\section{Acknowledgments}

This work was in collaboration with a number of colleagues. In particular, I am indebted to Wally Melnitchouk, Tony Thomas, Nobuo Sato, Joshua McKenney, Ping Wang, Xuangong Wang, Yusupujiang Salamu, Ben Bakker, Ziyue Li, Bailing Ma, Murat An and Alfredo Suzuki. I also thank Nico Stefanis and other organizers for this stimulating conference. This work was supported by the US DOE Contract No. DE-FG02-03ER41260.

\section{References}

[1] P.A.M.Dirac, Rev. Mod. Phys. 21: 392-399 (1949).

[2] S.Fubini, A.J.Hanson and R.Jackiw, Phys. Rev. D 7, 1732 (1973).

[3] L.Ya.Glozman, W.Plessas, K.Varga, and R.F.Wagenbrunn, Phys. Rev. D 58, 094030 (1998).

[4] R.F.Wagenbrunn, S.Boffi, W.Klink, W.Plessas and M.Radici, Phys.Lett. B 511, 33 (2001).

[5] R.F.T.Melde, K.Berger, L.Canton, W.Plessas and R.F.Wagenbrunn, Phys.Rev. D 76, 074020 (2007). 
[6] K. Hornbostel, Phys.Rev. D 45, 3781-3801 (1992).

[7] C.-R. Ji and C. Mitchell Phys.Rev. D 64, 085013 (2001).

[8] C.-R. Ji and A.T. Suzuki, Phys. Rev. D 87, 065015 (2013).

[9] C.-R. Ji, Z.Li and A.T. Suzuki, Phys. Rev. D 91:065020 (2015).

[10] Z. Li, M. An and C.-R. Ji, Phys. Red. D 92, 105014 (2015).

[11] S. Weinberg, Phys.Rev. 150, 1313 (1966).

[12] A.W. Thomas, W. Melnitchouk, F.M. Steffens, Phys. Rev. Lett. 85, 2892 (2000).

[13] W. Detmold, W. Melnitchouk, J.W. Negele, D.B. Renner, A.W. Thomas, Phys. Rev. Lett. 87, 172001 (2001).

[14] J.W. Chen, X. Ji, Phys. Lett. B 523, 107 (2001).

[15] D. Arndt, M.J. Savage, Nucl. Phys. A 697, 429 (2002).

[16] A.W. Thomas, Adv. Nucl. Phys. 13, 1-137 (1984).

[17] For review see X. Ji, Ann. Rev. Nucl. Part. Sci. 54, 413-450 (2004); A.V. Belitsky and A.V. Radyushkin, Phys. Rept. 418, 1-387 (2005).

[18] Y. Salamu, C.-R. Ji, W. Melnitchouk, and P. Wang, Phys. Rev. Lett. 114, 122001 (2015).

[19] M. Arneodo et al., Phys. Rev. D 50, 1 (1994).

[20] K. Ackerstaff et al., Phys. Rev. Lett. 81, 5519 (1998).

[21] A. Baldit et al., Phys. Lett. B 332, 244 (1994).

[22] R. S. Towell et al., Phys. Rev. D 64, 052002 (2001).

[23] J.R. McKenney, N. Sato, W. Melnitchouk and C.-R. Ji, Phys. Rev. D93, 054011 (2016).

[24] X. Wang, C.-R. Ji, W. Melnitchouk, Y. Salamu, A.W. Thomas, and P. Wang, Phys. Lett. B 762, 52 (2016); Phys. Rev. D, in press.

[25] B.L.G. Bakker, M.A. DeWitt, C.-R. Ji, Y. Mishchenko, Phys. Rev. D 72, 076005 (2005).

[26] J. D. Sullivan, Phys. Rev. D 5, 1732 (1972).

[27] C.-R. Ji, W. Melnitchouk and A. W. Thomas, Phys. Rev. Lett. 110, 179101 (2013),

[28] M. Burkardt, K.S. Hendricks, C.-R. Ji, W. Melnitchouk and A.W. Thomas, Phys. Rev. D 87, 056009 (2013).

[29] C.-R. Ji, W. Melnitchouk and A. W. Thomas, Phys. Rev. D 88, 076005 (2013).

[30] C.-R. Ji, W. Melnitchouk and A.W. Thomas, Phys. Rev. D 80, 054018 (2009).

[31] S.-J. Chang and T.-M. Yan, Phys. Rev. D 7, 1147 (1973).

[32] M. Alberg and G. Miller, Phys. Rev. Lett. 108, 172001 (2012).

[33] S. Chekanov, et al. Nucl. Phys. B637, 3 (2002).

[34] F.D. Aaron, et al. Eur. Phys. J. C 68, 381 (2010).

[35] S. Chekanov, et al. Nucl. Phys. B776, 1 (2007).

[36] C. Keppel, B. Wojtsekhowski, P. King, D. Dutta and J. Annand, as spokespersons: Measurement of Tagged Deep-Inelastic Scattering (TDIS), Jefferson Lab experiment PR12-15-006.

[37] N. Baillie, et al. Phys. Rev. Lett. 108, 142001 (2012).

[38] J. Badier, et al., Z. Phys. C 18, 281 (1983).

[39] B. Betev, et al., Z. Phys. C 28, 15 (1985).

[40] J.S. Conway, et al., Phys. Rev. D 39, 92 (1989).

[41] P. Jimenez-Delgado, W. Melnitchouk, and J. F. Owens, J. Phys. G 40, 093102 (2013).

[42] S. Forte, and G. Watt, Ann. Rev. Nucl. Part. Sci. 63, 291 (2013).

[43] G. P. Zeller et al., Phys. Rev. Lett. 88, 091802 (2002). 